\title{
IDENTIFICAÇÃO DE ADULTERANTES DE BIODIESEL METÍLICO DE CRAMBE EM MISTURAS COM DIESEL, USANDO FT-MIR E CARTAS DE CONTROLE MULTIVARIADAS BASEADAS NO SINAL ANALÍTICO LÍQUIDO
}

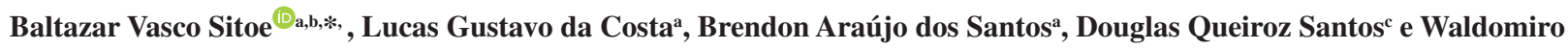 \\ Borges Neto ${ }^{a}$ \\ anstituto de Química, Universidade Federal de Uberlândia, 38400-902 Uberlândia - MG, Brasil
}

${ }^{b}$ Faculdade de Ciências Naturais e Matemática, Universidade Púnguè, 323 Chimoio - Manica, Moçambique

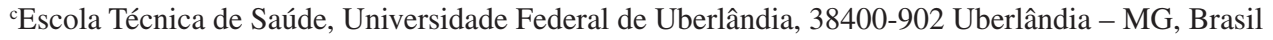

Recebido em 30/03/2019; aceito em 07/08/2019; publicado na web em 14/01/2020

\begin{abstract}
MONITORING OF ADULTERANTS IN CRAMBE METHYL BIODIESEL IN MIXTURES WITH DIESEL, USING FT-MIR AND MULTIVARIATE CONTROL CHARTS BASED ON NET ANALYTE SIGNAL. Adulterants in biodiesel/diesel blends modifies its physical and chemical properties. In this work, Fourier Transform Mid-Infrared spectrometry (FT-MIR) and multivariate control charts based on Net Analyte Signal (NAS) were used to monitor the quality of Crambe methyl biodiesel in relation to the biodiesel content and the presence of adulterants. The calibration model was constructed from the decomposition of the instrumental signals of the calibration samples into three vectors: NAS, interference and residual. From these vectors, three control charts were built: (i) NAS chart to monitor the analyte of interest (Crambe methyl biodiesel); (ii) Interference chart to monitor the data matrix (all components in diesel without the analyte of interest); and (iii) Residual chart to monitor any non-systematic variations. To validate the calibration model, other groups of B10, BX and B10 samples adulterated by direct addition of soybean oil and used fry oil in the range of $4.85-30.13 \%$ (v/v) were used. All samples prepared in-quality specifics were correctly classified as "in-quality control" samples, and other samples were correctly classified as "out-of-quality control" samples. Therefore, the methodology developed proved to be efficient in monitoring the quality of this fuel and it can be used by supervisory and quality control agencies.
\end{abstract}

Keywords: adulterants; Crambe biodiesel; MIR spectrometry; multivariate control charts; chemometrics.

\section{INTRODUÇÃO}

Atualmente, estudos relatam que o uso de combustíveis alternativos, principalmente os derivados de fontes renováveis, como o biodiesel, podem reduzir significativamente os problemas ambientais causados, pois minimizam os danos nos motores a diesel e as emissões de gases de efeito estufa, dependendo da matéria-prima utilizada. O biodiesel é um combustível produzido a partir da reação de esterificação e/ou transesterificação de gorduras animais ou de óleos vegetais com um álcool de cadeia curta, geralmente etanol ou metanol, em meio ácido, básico ou enzimático. ${ }^{1,2}$ É um combustível renovável, biodegradável, menos tóxico pela ausência de compostos sulfurados e aromáticos voláteis. Além disso, as propriedades físico-químicas do biodiesel são semelhantes ao diesel derivado de petróleo. ${ }^{3-8}$

Cerca de $82 \%$ do biodiesel usado no Brasil é produzido a partir do óleo de soja, pois o país possui maior potencial agrícola de produção desse cereal em quase todas as estações do ano. ${ }^{6,9}$ No entanto, o consumo familiar interno do óleo de soja e a exportação internacional deste cereal e do seu farelo para a produção de carnes impactam a produção do biodiesel a partir do óleo de soja. Por outro lado, o uso de óleos comestíveis para a produção do biodiesel, embora seja permitido pelo governo brasileiro, concorre com sua produção destinada para a alimentação humana e pode provocar o aumento de seus preços. Por isso, é necessário encontrar outros tipos de matérias primas para a produção do biodiesel que não participem da cadeia alimentar humana e que possam ser produzidos de acordo com as condições dos solos e climáticas do Brasil, como o é o caso do Crambe.,10

O Crambe (Crambe abyssinica) é uma cultura pertencente à família das crucíferas, originária da região do Mediterrâneo (nativa

*e-mail: baltazarvascositoe@yahoo.com.br da Etiópia e da Tanzânia). No entanto, também é capaz de se adaptar às mais diversas condições climáticas (regiões frias e secas), sendo resistente a pragas e doenças; tem um grande potencial para a produção do biodiesel devido ao seu curto ciclo anual, entre 90 a 100 dias, possibilitando o seu uso como segunda safra, ou seja, na rotação de culturas. No Brasil, ele é cultivado no sul e sudeste; possui um ciclo de cultivo muito reduzido, rusticidade, bom potencial produtivo e com baixos custos referentes à produção e ao cultivo mecanizado. A semente de Crambe apresenta alto teor de óleo, em torno de $38 \%$, que pode ser facilmente extraído por prensagem mecânica. ${ }^{11,12}$ Os frutos são ricos em ácidos graxos, linoleico, palmitoleico e erúcico (ácido graxo monoinsaturado), não sendo, por isso, adequados para a alimentação humana e animal. ${ }^{1,13-16}$ Em comparação a outras oleaginosas mais usadas para a produção do biodiesel (como canola, girassol e soja), os custos de produção associados ao Crambe são os mais baixos. ${ }^{17} \mathrm{O}$ óleo de Crambe apresenta um teor de $60 \%$ de ácidos graxos monoinsaturados e $10 \%$ de poli-insaturados, contra mais de $50 \%$ de poli-insaturados encontrados na soja. O óleo de soja é mais suscetível à oxidação devido ao maior número de ácidos poli-insaturados e, por consequência, o biodiesel de soja gerado também seja menos estável que o de Crambe. ${ }^{2} \mathrm{O}$ biodiesel de Crambe tem o mesmo consumo e eficiência na conversão energética específica aos motores de óleo diesel e com uma redução significativa de emissão de gases como o monóxido de carbono. Por isso, Crambe abyssinica constitui uma fonte promissora para a produção do biodiesel. ${ }^{1,9,10,15}$

Como o biodiesel tem propriedades físico-químicas semelhantes ao do óleo diesel, ele pode ser usado na sua forma pura sem modificações nos motores de ciclo diesel, ou pode ser misturado com o óleo diesel. ${ }^{18} \mathrm{O}$ biodiesel usado na sua forma pura é denominado B100 e em misturas com o diesel $\mathrm{BX}$, em que $\mathrm{X}$ corresponde à proporção percentual volumétrica do biodiesel na mistura. A Agência Nacional 
do Petróleo, Gás Natural e Bicombustíveis (ANP) estabeleceu que as misturas biodiesel/diesel atualmente usadas no Brasil são B10, ou seja, misturas constituídas por do biodiesel em de diesel a partir de $1^{\circ}$ de março de 2018. ${ }^{19}$ Mas essas misturas podem não corresponder a B10, ou podem sofrer adulterações por adição ilegal de óleos vegetais pelos comerciantes com fins lucrativos ilícitos. Portanto, é importante garantir a qualidade dessas misturas de acordo com os padrões e regulamentos de qualidade.

O monitoramento da qualidade dos combustíveis é importante, não somente em função do desempenho dos motores de automóveis, mas devido ao impacto ambiental das emissões de poluentes. Combustíveis contaminados ou adulterados podem provocar superaquecimento e aceleração excessiva do motor, além de aumentarem o consumo do combustível, a emissão de material particulado e de gases de exaustão. ${ }^{20-23} \mathrm{~A}$ contaminação de combustíveis pode ocorrer por transesterificação incompleta dos óleos ou misturas com outros combustíveis durante o transporte, enquanto que adulteração pode ocorrer por adição de um produto mais barato com propriedades físico-químicas semelhantes ao biodiesel e ao diesel, visando a obtenção de lucros ilícitos. A adição de óleo vegetal às misturas biodiesel/diesel é uma das adulterações mais fáceis e usuais devido ao óleo vegetal possuir boa miscibilidade ao combustível biodiesel/diesel. ${ }^{24}$ Assim, é necessário e importante usar métodos analíticos para detectar a presença de adulterantes. De acordo com a Agência Nacional de Petróleo Gás e Biocombustível (ANP), os métodos padrão, como EN $14078^{25} \mathrm{e}$ ASTM D7371-07, ${ }^{26}$ utilizam a espectrometria no infravermelho médio (MIR) com métodos univariados e multivariados, respectivamente, para quantificar o teor do biodiesel em misturas com o diesel. ${ }^{3}$ Mas métodos qualitativos, como a combinação da espectrometria MIR e cartas de controle multivariadas, também podem ser usados para o monitoramento do teor do biodiesel e de adulterantes durante o processo de produção ou em análises de rotina. Esses métodos, além de serem rápidos, não são destrutivos, fazem análises in situ e têm vantagem do uso de poucas amostras para a calibração e validação. ${ }^{27,28}$

O desenvolvimento de novos métodos analíticos automatizados, in-line e rápidos para monitorar a qualidade do teor das misturas biodiesel/diesel e a presença de adulterantes é de extrema importância para pesquisas, com vista a desenvolver a utilização de instrumentos que sejam capazes de fornecer resultados para diversos parâmetros de qualidade de forma rápida. ${ }^{29} \mathrm{~A}$ maioria das pesquisas desenvolvidas e o maior número de aplicações usando as técnicas espectrométricas referem-se às transições vibracionais e rotacionais nas ligações das moléculas que ocorrem na região do infravermelho médio, porque nessa região do espectro (principalmente na região de "impressão digital"), ocorrem, essencialmente, transições fundamentais, em que pequenas diferenças na estrutura e na constituição de uma molécula resultam em mudanças significativas no perfil e na distribuição das bandas de absorção. Por isso, simples semelhanças ou diferenças entre dois espectros nesta região, bem como nas outras, constituem fortes evidências da identidade dos compostos que produzem os espectros. ${ }^{30,31}$ As cartas de controle multivariadas baseadas no sinal analítico líquido (NAS - do inglês, Net Analyte Signal) são utilizadas no monitoramento simultâneo de qualidade de mais de uma variável de um determinado produto ou processo de produção. ${ }^{32}$ Nesse caso, o uso das cartas de controle multivariadas e espectrometria MIR para o monitoramento da qualidade das misturas biodiesel/diesel é vantajoso, pois esse método monitora simultaneamente a qualidade de todos os componentes de cada amostra em uma mesma análise.

Existem trabalhos publicados usando cartas de controle multivariadas baseadas no NAS e técnicas espectrométricas para monitorar a qualidade das composições polimórficas de formulações farmacêuticas, ${ }^{32-34}$ entre outros processos estatísticos de produção. ${ }^{35,36}$ Porém, trabalhos publicados para monitorar o teor do biodiesel e a presença de adulterantes nas misturas biodiesel/diesel usando cartas de controle multivariadas e espectrometria MIR são muito escassos. ${ }^{37-39}$ Além disso, ainda não existem trabalhos publicados para identificar adulterantes no biodiesel metílico de Crambe usando este método. Por isso, o objetivo deste trabalho foi construir e validar modelo de cartas de controle multivariadas baseadas no NAS aliadas à espectrometria MIR para monitorar a qualidade de misturas B10 do biodiesel metílico de Crambe em relação ao teor e à adição direta de adulterantes (óleo de soja e óleo de fritura residual).

\section{MATERIAIS E MÉTODOS}

\section{Produção do biodiesel}

O biodiesel usado para a preparação de amostras foi sintetizado no Laboratório de Biocombustíveis do Instituto de Química da Universidade Federal de Uberlândia - Minas Gerais, Brasil. Esta síntese foi feita em duas etapas: a esterificação e a transesterificação. A esterificação (catálise ácida) foi realizada para eliminar impurezas e converter os ácidos graxos livres presentes no óleo do Crambe e parte dos triglicerídeos em ésteres metílicos, antes da etapa de transesterificação. ${ }^{1,40}$ Nesse caso, a proporção mássica do metanol para o óleo foi mantida na proporção de $1: 1(\mathrm{~m} / \mathrm{m})$. Primeiro, adicionou-se $1 \%(\mathrm{~m} / \mathrm{m}$, em relação a massa do óleo) do ácido sulfúrico concentrado, usado como catalisador ao álcool e, posteriormente, a mistura resultante foi adicionada ao óleo de Crambe num balão de vidro de fundo redondo. A mistura total permaneceu sob refluxo em ebulição durante $3 \mathrm{hs} \mathrm{a} 60{ }^{\circ} \mathrm{C}$ numa agitação magnética a $600 \mathrm{rpm}$. Após a reação de esterificação, a mistura heterogênea resultante foi transferida para um funil de separação e permaneceu em repouso durante 24 hs para separar o excesso de álcool, ácido sulfúrico e quaisquer outras impurezas presentes na mistura. ${ }^{40-42}$

Na etapa da transesterificação (catálise básica), o óleo resultante da esterificação foi submetido a uma reação com o metanol, e com o hidróxido de potássio como catalisador, à pressão atmosférica. A proporção molar do óleo e álcool foi de $6: 1$ e $1 \%$ (m/m, em relação à massa do óleo) do hidróxido de potássio. $\mathrm{O}$ catalisador foi inicialmente dissolvido no álcool metílico e, depois, a mistura resultante foi adicionada ao óleo. A reação de transesterificação foi mantida a $65^{\circ} \mathrm{C}$ durante $2 \mathrm{~h}$ e em agitação magnética a $450 \mathrm{rpm}$. Após a reação, a solução resultante foi depositada em um funil de separação e mantido em repouso por $24 \mathrm{~h}$ para separar o glicerol e impurezas (camada inferior) do biodiesel (camada superior). A camada inferior, que continha impurezas e glicerol, foi separada e guardada em recipiente para posterior tratamento ambiental, enquanto a camada superior (biodiesel) foi submetida a lavagens sucessivas com água deionizada quente a $50{ }^{\circ} \mathrm{C}$ até não se observar alteração no $\mathrm{pH}$ da água de lavagem. Depois disso, o biodiesel foi submetido a uma rota-evaporação durante uma hora a $88 \mathrm{rpm}$ para remover o excesso de água e de metanol. Finalmente, foi secado, utilizando-se o sulfato de sódio anidro e filtrado sob vácuo em papel de filtro de separação. . $^{1,243}$ Para se obter maior representatividade e aumentar a robustez dos modelos quimiométricos, foram preparados 20 lotes de B100.

\section{Preparação de amostras}

Para a preparação de amostras das misturas biodiesel/diesel, 18 lotes do óleo diesel puro foram providenciados pela indústria Transpetro S/A, sediada na cidade de Uberlândia. Para a construção de modelos de calibração e de validação das cartas de controle multivariadas, foram preparados sete conjuntos de amostras (I, II, III, IV, V, VI e VII). Todas as amostras foram pesadas em uma balança analítica (sartorius, modelo BP211D). As frações mássicas (\% m/m) dos componentes das 
amostras foram convertidas em frações volumétricas (\% v/v) através da densidade, com a finalidade de expressar os resultados para teor de biodiesel na mesma unidade de medida referenciada nas normas EN 14078 e ABNT NBR $15568 .{ }^{44}$ As soluções resultantes de todas as amostras de calibração e de validação foram homogeneizadas em agitador Vortex por pelo menos um minuto.

No uso do método de cartas de controle multivariadas, o tamanho de cada conjunto de amostras não é estabelecido por nenhuma norma, porém, costuma-se definir o número de amostras que sejam representativas de cada conjunto dentro de uma faixa de concentração desejável para a modelagem. ${ }^{32-36}$

O conjunto I de amostras foi constituído por 18 amostras do diesel puro, designados por amostras do branco ou placebo (apenas amostras do diesel, sem o analito de interesse), em que tais amostras foram usadas para a construção do espaço interferente. $\mathrm{O}$ conjunto II foi constituído por 21 amostras B10, designado por amostras de calibração ou amostras "dentro do controle de qualidade", isso é, amostras que foram preparadas dentro das especificações de qualidade e sob condições normais de operação (NOC - do inglês, Normal Operation Condition). ${ }^{32}$ Elas foram preparadas com a variação de $\pm 0.5 \%$ permitida pela $\mathrm{ANP},{ }^{19}$ ou seja, com teor de biodiesel variando dentro das especificações de qualidade, de 9.50-10.49\% (v/v). Esse conjunto de amostras foi utilizado para calcular os limites estatísticos de cada carta e calibrar o modelo das cartas de controle multivariadas.

Os conjuntos III, IV e V são do primeiro caso de amostras de validação do modelo das cartas de controle multivariadas. Essa validação foi em relação ao teor do biodiesel em diesel. As amostras destes conjuntos não possuíam adulterantes na sua composição, isso é, elas tinham apenas misturas de biodiesel/diesel, mas com a variação do teor do biodiesel para validar o modelo de cartas de controle multivariadas já construído. O conjunto III foi constituído por amostras de validação com teor do biodiesel "dentro do controle de qualidade", B10; o conjunto IV consistiu em amostras de validação "fora do controle de qualidade" com teor do biodiesel abaixo do B10, variando na faixa de 4.80-9.30\% (v/v); e o conjunto $\mathrm{V}$ também foi constituído por amostras de validação "fora do controle de qualidade", mas com teor do biodiesel acima do B10, variando na faixa de $10.64-15.98 \%(\mathrm{v} / \mathrm{v})$.

Os conjuntos VI e VII referem-se ao segundo caso de amostras de validação do modelo das cartas controle multivariadas. Essas amostras foram preparadas da seguinte maneira: primeiro preparam-se as amostras B10; depois fez-se a adição direta de adulterantes (óleo de fritura residual (OFR) e óleo de soja), na faixa de concentração de 4.85-30.13\% (v/v), às misturas B10. Após a adição de adulterante na mistura, alterou tanto o teor do biodiesel como do diesel. A Tabela 1 apresenta o número total de amostras de cada conjunto, a função, as suas composições percentuais do biodiesel, do diesel e dos adulterantes.

\section{Aquisição dos dados espectrais}

Os dados espectrais MIR foram adquiridos em quintuplicatas na faixa de 4000-650, usando um equipamento Spectrum Two, equipado com um acessório amostrador de refletância total atenuada horizontal (HATR), com o cristal de ZnSe. Foi usada uma resolução de 4 e 16 varreduras. Foi aplicada correção de linha de base nos espectros. Os modelos de cartas de controle multivariadas foram construídos utilizando o software MATLAB versão 7.5 e PLS_Toolbox8.7, usando rotinas desenvolvidas para o ambiente MATLAB.

\section{Aplicação da quimiometria e espectrometria no infravermelho médio para construção do modelo de cartas de controle multivariadas baseadas no sinal analítico líquido}

A metodologia detalhada da construção de cartas de controle multivariadas baseadas no NAS está presente na literatura. ${ }^{32,38}$ Neste trabalho, a construção do modelo quimiométrico de cartas de controle multivariadas baseadas no NAS foi feita através da decomposição de espectros originais de amostras de calibração (como se pode ver na Figura 1) em três vetores: NAS, interferente e residual. Porém, na primeira fase, desenvolveu-se o espaço interferente, que descreveu todas as variações dos componentes da amostra. Este espaço foi construído usando espectros das amostras do branco ou placebo, aplicando a Análise de Componentes Principais (PCA - do inglês, Principal Component Analysis) de espectros de amostras do branco. $\mathrm{O}$ vetor interferente foi calculado através da projeção dos vetores espectrais de B10 no espaço interferente. Em seguida, definiu-se o vetor NAS, usando espectros das amostras com o analito de interesse, B10, chamadas amostras "dentro do controle de qualidade". O vetor residual foi calculado como a parte dos vetores espectrais não descrito pelos vetores NAS e interferente. Esse cálculo foi feito por uma simples subtração vetorial.

$\mathrm{O}$ vetor NAS fornece a informação do analito de interesse na amostra (biodiesel, neste trabalho); o vetor interferente caracteriza os

Tabela 1. Composição percentual de amostras de calibração e de validação em relação ao teor do biodiesel em diesel e em relação à presença de adulterantes por adição direta de óleos de soja e de fritura residual às misturas B10

\begin{tabular}{|c|c|c|c|c|c|c|}
\hline Conjunto & $\begin{array}{l}\text { Número de } \\
\text { amostras }\end{array}$ & Função & $\begin{array}{l}\text { Biodiesel } \\
(\% \mathrm{v} / \mathrm{v})\end{array}$ & $\begin{array}{l}\text { Diesel } \\
(\% \mathrm{v} / \mathrm{v})\end{array}$ & Adulterante & $\begin{array}{c}\text { Adulterante no B10 } \\
(\% \mathrm{v} / \mathrm{v})\end{array}$ \\
\hline I & 18 & $\begin{array}{l}\text { Construção do espaço } \\
\text { interferente }\end{array}$ & --- & 100 & --- & --- \\
\hline II & 21 & $\begin{array}{l}\text { Cálculo dos limites estatísticos e } \\
\text { calibração do modelo }\end{array}$ & $9.50-10.49$ & $89.51-90.50$ & --- & --- \\
\hline III & 11 & $\begin{array}{l}\text { Validação dentro do controle, } \\
\text { B10 }\end{array}$ & $9.62-10.40$ & $89.60-93.38$ & --- & --- \\
\hline IV & 10 & $\begin{array}{l}\text { Validação fora do controle, } \\
\text { abaixo de B10 }\end{array}$ & $4.80-9.30$ & $90.70-95.20$ & --- & --- \\
\hline V & 10 & $\begin{array}{l}\text { Validação fora do controle, acima } \\
\text { de B10 }\end{array}$ & $10.64-15.98$ & $84.02-89.36$ & --- & --- \\
\hline VI & 20 & $\begin{array}{l}\text { Validação com B10 adulterado } \\
\text { por óleo de soja }\end{array}$ & $4.85-8.87$ & $65.10-76.18$ & Óleo de soja & $4.95-30.05$ \\
\hline VII & 20 & $\begin{array}{l}\text { Validação com B10 adulterado } \\
\text { por OFR }\end{array}$ & $4.86-6.65$ & $65.01-88.37$ & OFR & $4.98-30.13$ \\
\hline
\end{tabular}


constituintes dos interferentes (diesel puro); e o vetor residual explica todas as informações não contidas nos vetores NAS e interferente. O NAS é a parte do espectro que é ortogonal ao espaço interferente. No entanto, como existem muitas direções ortogonais a este espaço, definiu-se uma direção específica do NAS, ou seja, a direção NAS, para o analito de interesse, usando vetores espectrais de B10. Para reduzir o ruído desses vetores, essa direção foi definida no espaço multivariado, calculando-se um vetor médio, chamado vetor de regressão NAS. ${ }^{45}$

Os limites estatísticos das cartas NAS, interferente e residual foram respectivamente calculados a partir dos vetores NAS, interferente e residual dos espectros das amostras de calibração, B10. Posteriormente, esses limites foram plotados dentro do modelo de calibração das cartas de controle multivariadas, conforme ilustrado na Figura 2 (os limites de cada carta são apresentados em linha tracejada). Para calcular os limites da carta NAS, projetou-se cada espectro de amostras de calibração ao vetor de regressão NAS. Essa projeção forneceu um valor escalar de cada amostra proporcional à concentração do analito de interesse, chamado valor NAS. A carta NAS apresenta dois limites, um superior e outro inferior, porque foram usados $95 \%$ de confiança, a média e o desvio padrão dos valores NAS, conforme a Equação 1:46

$$
\operatorname{Limit}_{95 \%}=\overline{\mathbf{n a s}}_{\mathrm{NOC}} \pm 2 \mathrm{~S}_{\mathrm{NOC}}
$$

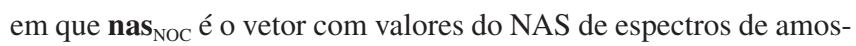
tras "dentro do controle de qualidade" e $\left(\mathrm{S}_{\mathrm{NOC}}\right)$ é o desvio padrão dos nas NOC $_{\text {. }}$

O limite da carta interferente foi calculado utilizando $d$-estatístico. Para tal, os espectros de amostras de calibração foram projetados numa região do espaço interferente construído a partir das amostras do branco ou placebo; calcularam novas coordenadas para os vetores interferentes em um sistema de eixos denominado matriz scores. Em seguida, calculou-se o valor de $d$-estatístico definido para cada sinal espectral de amostras de calibração, ou seja, calculou-se a distância das amostras ao centro do modelo PCA. $\mathrm{O} d$-estatístico, multiplicado por uma constante, seguiu uma distribuição $F \operatorname{com} A$ e $\left(I_{\mathrm{NOC}}-A\right)$ graus de liberdade, em que $A$ é o número de componentes principais usados no modelo PCA e $I_{\mathrm{NOC}}$ o número de amostras de calibração. Assim, o limite de $95 \%$ de confiança para a carta interferente foi calculado usando a seguinte Equação $2:{ }^{47}$

$$
D_{\lim , 0.95}=F_{0.95}\left(A, I_{\mathrm{NOC}}-A\right)\left(\frac{\mathrm{A}\left(I_{\mathrm{NOC}}{ }^{2}-I_{\mathrm{NOC}}\right)}{I_{\mathrm{NOC}}\left(I_{\mathrm{NOC}}-\mathrm{A}\right)}\right)
$$

O limite da carta residual foi calculado baseando-se nos valores Q-estatísticos dos vetores residuais, calculados a partir da matriz contendo os vetores interferentes e da matriz contendo os vetores NAS. Assim, os valores $Q$-estatísticos foram calculados da soma dos quadrados dos resíduos, cuja média corresponde ao limite da carta residual, conforme a Equação 3:46

$$
\mathbf{Q}_{\mathrm{NOC}}=\mathbf{R}_{\mathrm{RES}}^{\mathrm{T}} \mathbf{R}_{\mathrm{RES}} \approx \mathrm{g} X_{\mathrm{h}}^{2}
$$

em que o $\mathbf{Q}_{\mathrm{NOC}}$ contém os valores $Q$-estatísticos de espectros das amostras "dentro do controle de qualidade" na diagonal, $\mathbf{R}_{\mathrm{RES}}$ são vetores residuais das amostras de calibração. O $Q$-estatístico para novas amostras seguiu uma distribuição de qui-quadrado, em que o fator de escalamento "g" e os graus de liberdade "h" são chamados de encontros da distribuição. O limite de confiança para a carta residual é calculado conforme as equações 4,5 e 6 . Para as amostras consideradas "dentro do controle de qualidade", os vetores residuais representam variações apenas provenientes do ruído instrumental do equipamento. Se o vetor residual de uma nova amostra não for apenas o ruído instrumental (aleatório), o valor de $Q$-estatístico será maior e a amostra irá localizar-se fora (acima) do limite da carta residual: ${ }^{47,48}$

$$
Q_{\alpha}=\theta_{1}\left[\frac{c_{\alpha} \sqrt{2 \theta_{2} h_{0}^{2}}}{\theta_{1}}+1+\frac{\theta_{2} h_{0}\left(h_{0}-1\right)}{\theta_{1}^{2}}\right]^{\frac{1}{h_{0}}}
$$

em que $c_{\alpha}$ é o desvio padrão normal; $\theta_{i}$ são as raízes características calculadas pela Equação 5:

$$
\theta_{i}=\sum_{j=k+1}^{n} \lambda_{j}^{i} \mathbf{i}: 1,2 \text { e } 3
$$

sendo $k$ o número de componentes principais $(\mathrm{PCs})$ retidos no modelo e $n$ o número total de PCs; $h_{0}$ definido pela Equação 6:

$$
h_{0}=1-\left(\frac{2 \theta_{1} \theta_{3}}{3 \theta_{2}^{2}}\right)
$$

Finalmente, os valores NAS, $d$-estátistico e $Q$-estatístico calculados foram plotados, respectivamente, nas cartas NAS, Interferente e Residual. Assim, uma amostra é considerada "dentro do controle de qualidade" se ela estiver dentro dos limites estatísticos das três cartas (Figura 2). Se ela estiver fora dos limites de pelo menos uma das cartas é considerada amostra "fora do controle de qualidade".

\section{RESULTADOS E DISCUSSÃO}

\section{Construção do modelo de cartas de controle multivariadas}

Usando os espectros das amostras do branco, foi construído o espaço interferente do modelo com duas componentes principais que explicaram $99.98 \%$ da variância total dos dados. A Figura 1 apresenta os espectros de amostras das misturas B10 (cor vermelha), usados para o cálculo dos limites estatísticos e calibração do modelo. Os espectros destas amostras foram corrigidos pela técnica da linha de base, depois calculou-se o vetor de regressão NAS e fez-se a decomposição dos seus espectros em vetores NAS (cor preta), interferente (cor azul) e residual (cor lilás). Os vetores NAS correspondem à contribuição do biodiesel nas amostras; os vetores interferentes, que correspondem às contribuições do diesel, apresentaram bandas de absorção com maiores intensidades que as bandas dos vetores NAS. Esse caso deveu-se à maior concentração do diesel existente nestas amostras; os vetores residuais apresentaram-se com valores de intensidade aleatórios e muito menores aos apresentados pelos vetores NAS e interferente, porque, nas amostras de calibração, a parte não modelada pelos outros dois vetores refere-se apenas ao ruído instrumental, cujas principais características são a sua baixa intensidade e a variação aleatória. ${ }^{32,45}$

A partir dos vetores NAS, interferente e residual, e usando $95 \%$ de confiança, foram calculados os limites estatísticos de cada carta de controle. Esses limites estão apresentados na Tabela 2. Os valores dos limites estatísticos de cada carta são proporcionais às intensidades dos seus vetores, ou seja, o limite da carta interferente é maior que os limites das cartas NAS e residual; o da carta residual é menor que os limites das cartas NAS e interferente.

Esses limites estatísticos foram plotados nas respectivas cartas, conforme é apresentado na Figura 2; os pontos ( ) representam os valores escalares NAS, os valores D e os valores Q para cada uma das amostras de calibração, no eixo das ordenadas; no eixo das abscissas representam as amostras de calibração.

Assim, todas as amostras de calibração são consideradas amostras "dentro do controle de qualidade" ou dentro das especificações de 

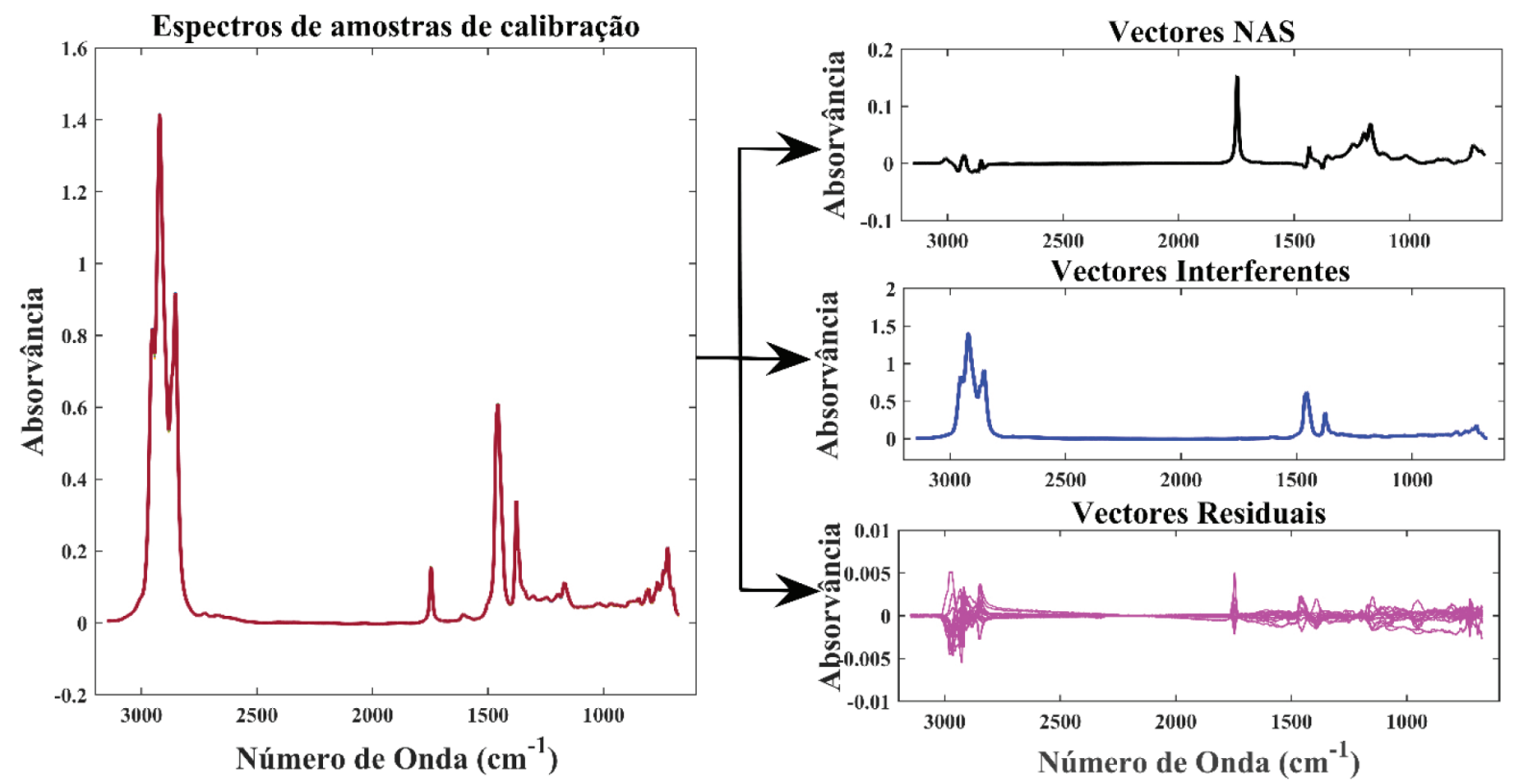

Figura 1. Decomposição de espectros de amostras de calibração em vetores NAS, interferentes e residuais

Tabela 2. Valores dos limites estatísticos das cartas NAS, interferente e residual

\begin{tabular}{cccc}
\hline \multicolumn{2}{c}{ Carta NAS } & Carta Interferente & Carta Residual \\
\hline Limite inferior & Limite Superior & Valor D & Valor Q \\
\hline $3.0 \times 10^{-3}$ & $3.4 \times 10^{-3}$ & 4.69 & $1.24 \times 10^{-5}$ \\
\hline
\end{tabular}

qualidade, porque todas elas estão dentro dos limites de cada uma das três cartas. Caso contrário, isso é, se uma amostra estivesse fora do limite de pelo menos uma das cartas, ela seria considerada como amostra "fora do controle de qualidade".

Validação do modelo de cartas de controle multivariadas em relação ao teor do biodiesel em diesel

Os resultados de validação do modelo de cartas de controle multivariadas em relação ao teor do biodiesel nas misturas biodiesel/ diesel são apresentados na Figura 3. Nessa validação, verificou-se que as amostras de calibração e de validação dentro do controle têm os seus valores NAS, D e Q dentro dos limites estatísticos das cartas NAS, interferente e residual, respetivamente, e, portanto, todas as amostras foram corretamente classificadas como amostras "dentro do controle de qualidade", o que está de acordo com o esperado. Na validação com as amostras do conjunto IV, como o teor do biodiesel nas amostras é menor que $9.50 \%$ (v/v), verificando a carta NAS, notou-se que todas as amostras apresentaram os valores NAS abaixo do limite inferior e de forma decrescente de acordo com os valores do teor do biodiesel apresentados na Tabela 1. Isso aconteceu porque a diferença entre os valores NAS previstos e de referência está abaixo do valor do limite inferior da carta NAS; já as amostras do conjunto $\mathrm{V}$, como elas têm teor do biodiesel maior que $10.50 \%(\mathrm{v} / \mathrm{v})$, apresentaram os valores NAS de forma crescente e acima do limite superior da carta NAS.
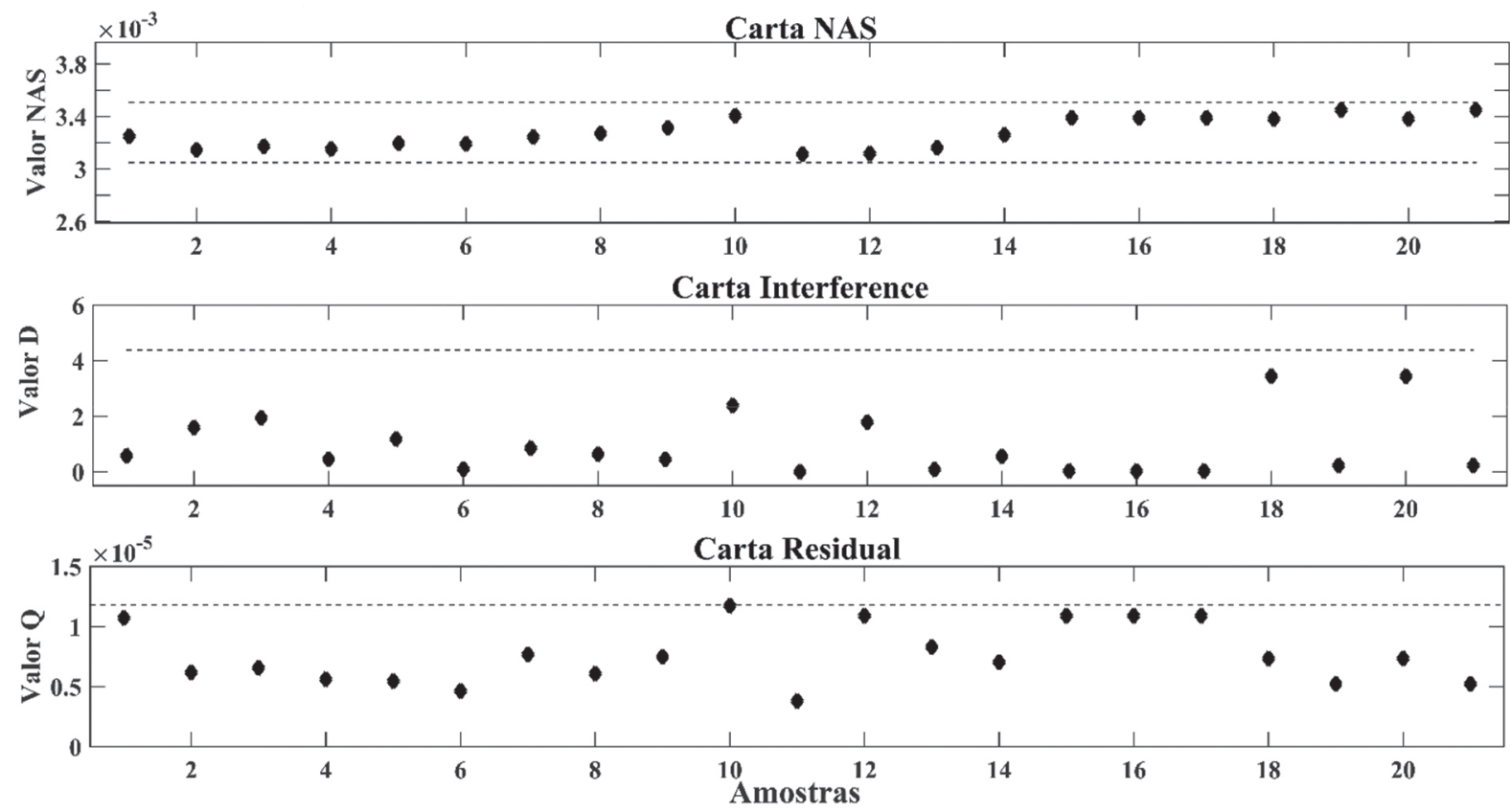

Figura 2. Modelo de calibração das cartas de controle multivariadas com amostras de calibração (•) 

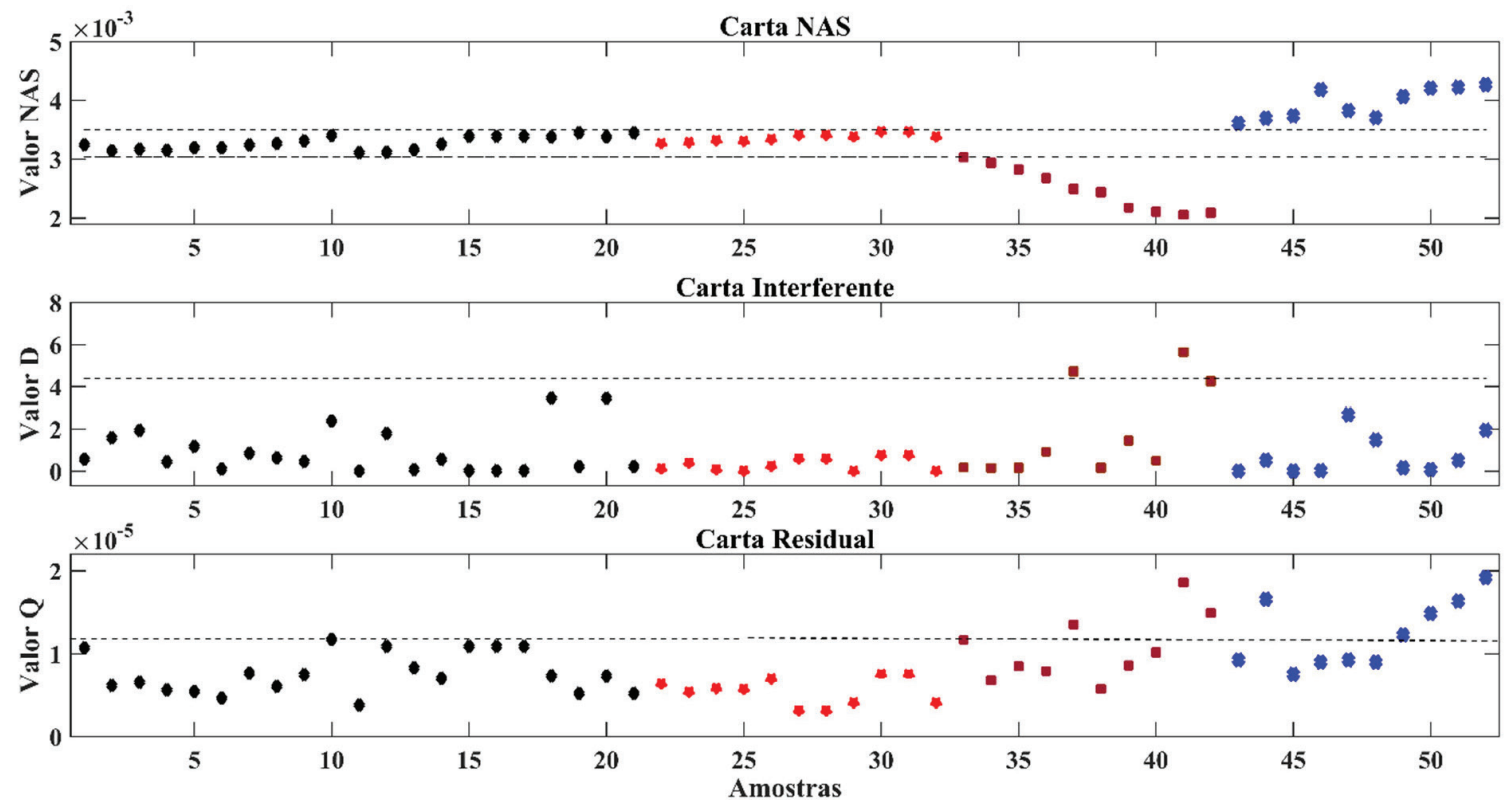

Figura 3. Modelo de validação das cartas de controle multivariadas com amostras de: calibração ( $\bullet$ ); validação dentro de controle ( $\star$ ); validação fora do controle com menor teor do biodiesel ( $\mathbf{\square})$; e validação fora do controle com maior teor do biodiesel (*)

Era esperado que as amostras dos conjuntos IV e V apresentassem os valores D e Q acima dos limites estatísticos das cartas interferente e residual, respectivamente, porque a variação do teor do biodiesel influenciou no de diesel. No entanto, algumas amostras apresentaram os seus valores $\mathrm{D}$ e $\mathrm{Q}$ dentro dos limites estatísticos. Isso pode ser explicado supondo que parte dos valores D e Q destas amostras foram modelados pela carta NAS. ${ }^{33}$ Contudo, todas as amostras dos conjuntos IV e V foram corretamente classificadas como amostras "fora do controle de qualidade", pois todas elas ficaram fora dos limites estatísticos de pelo menos uma das cartas.

Assim, o modelo de cartas de controle multivariadas mostrou-se eficiente para monitorar outras amostras em relação ao teor do biodiesel no diesel, porque as amostras de validação com o teor do biodiesel dentro das especificações de qualidade foram corretamente classificadas como amostras "fora do controle de qualidade", e as amostras com o teor do biodiesel abaixo ou acima das especificações de qualidade foram corretamente classificadas como amostras "fora do controle de qualidade".

Validação do modelo de cartas de controle multivariadas em relação à presença de adulterantes

A outra forma de validar o modelo de cartas de controle multivariadas foi feita através das amostras dos conjuntos VI e VII. Essas amostras foram preparadas através da adição direta de adulterantes, óleo de soja e OFR, às misturas B10 previamente preparadas. Essa adição de adulterantes alterou tanto o teor do biodiesel como o do diesel nas misturas (vide a Tabela 1).

O modelo de cartas de controle multivariadas validado pelos conjuntos de amostras adulteradas é apresentado na Figura 4. Nesse modelo, todas as cartas de controle (NAS, interferente e residual) foram capazes de distinguir a presença de amostras adulteradas por
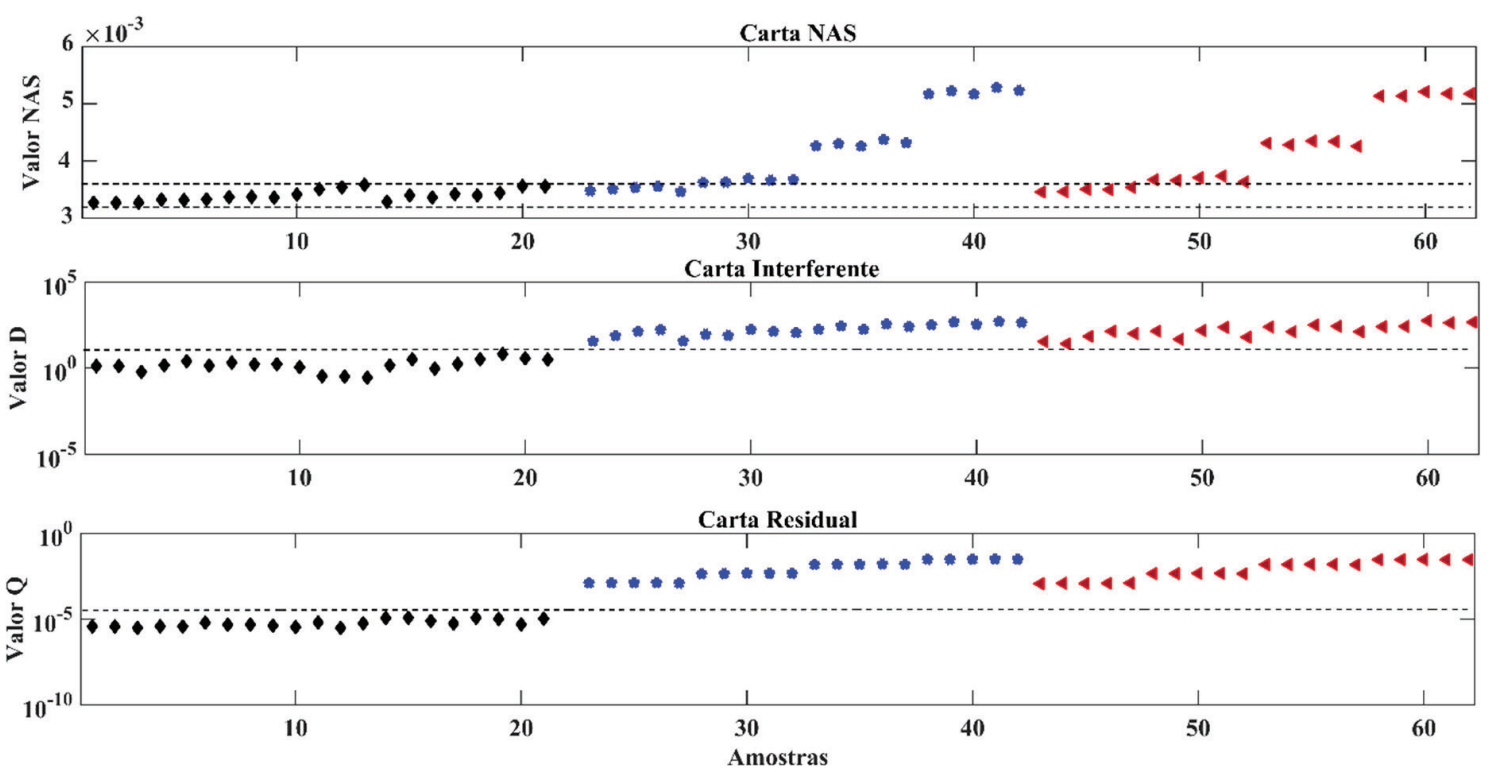

Figura 4. Modelo de validação das cartas de controle multivariadas com amostras de: calibração ( ); validação com B10 adulterado por óleo de soja ( $\star$ ); e validação com B10 adulterado por óleo de fritura residual $(<)$ 
óleo de soja e por óleo de fritura residual, porque todas as amostras extrapolaram os limites estatísticos das cartas.

Embora possa as primeiras cinco amostras adulteradas por óleo de soja e por óleo de fritura residual se encontrem "dentro do controle de qualidade" na carta NAS (porque estão dentro dos limites estatísticos desta carta), elas são consideradas como amostras "fora do controle de qualidade", porque extrapolaram os limites estatísticos de outras cartas (interferente e residual). Assim, todas as amostras dos conjuntos de validação por adição direta dos óleos de soja e de fritura residual foram corretamente classificadas como amostras "fora do controle de qualidade", pois todas elas extrapolaram os limites estatísticos das cartas. Portanto, ao monitorar a qualidade das amostras, é necessário que o analista observe os limites estatísticos de todas as cartas, pois basta a amostra sair em pelo menos um dos limites para ser considerada como amostra "fora do controle de qualidade", o que torna as cartas de controle multivariadas uma ferramenta poderosa para o controle de qualidade.

\section{CONCLUSÃO}

Neste trabalho, através do uso de espectrometria no infravermelho médio e cartas de controle multivariadas baseadas no NAS foi possível monitorar o teor do biodiesel no diesel e identificar a presença de adulterantes no biodiesel metílico de Crambe, B10. Todas as amostras de validação com o teor do biodiesel dentro das especificações de qualidade, B10, foram corretamente classificadas como amostras "dentro do controle de qualidade"; e as amostras de validação com o teor do biodiesel fora das especificações de qualidade (amostras com teor do biodiesel diferentes de B10) foram corretamente classificadas como amostras "fora do controle de qualidade". As amostras adulteradas por adição direta do óleo de soja e do óleo de fritura residual também foram corretamente classificadas como amostras "fora do controle de qualidade".

O método usado é rápido e eficiente, não é destrutivo e não necessita da preparação prévia da amostra. Por isso, esse método consiste em uma alternativa viável que pode ser usada pelos órgãos de fiscalização e de controle de qualidade desse combustível.

\section{AGRADECIMENTOS}

Os autores deste trabalho agradecem ao programa do Banco Mundial e Ministério da Ciência e Tecnologia, Ensino Superior e Técnico Profissional de Moçambique, processo número 24.06.2016, pelo apoio financeiro da bolsa de estudos, e ao Conselho Nacional de Desenvolvimento Científico e Tecnológico (CNPq:306287/2016-1).

\section{REFERÊNCIAS}

1. Silveira, E. V.; Vilela, L. S.; Castro, C. F. de S.; Lião, L. M.; Gambarra Neto, F. F.; de Oliveira, P. S. M.; Ind. Crops Prod. 2017, 97, 545.

2. Wazilewski, W. T.; Bariccatti, R. A.; Martins, G. I.; Secco, D.; Souza, S. N. M. de; Rosa, H. A.; Chaves, L. I.; Ind. Crops Prod. 2013, 43, 207.

3. Paiva, E. M.; Rohwedder, J. J. R.; Pasquini, C.; Pimentel, M. F.; Pereira, C. F.; Fuel 2015, 160, 57.

4. Achten, W. M. J.; Verchot, L.; Franken, Y. J.; Mathijs, E.; Singh, V. P.; Aerts, R.; Muys, B.; Biomass Bioenergy 2008, 32, 1063

5. Correia, R. M.; Domingos, E.; Cáo, V. M.; Araujo, B. R. F.; Sena, S.; Pinheiro, L. U.; Fontes, A. M.; Aquino, L. F. M.; Ferreira, E. C.; Filgueiras, P. R.; Romão, W.; Talanta 2018, 176, 26.

6. De Oliveira, F. C.; Coelho, S. T.; Renewable Sustainable Energy Rev. 2017, 75, 168.

7. Shen, X.; Shi, J.; Cao, X.; Zhang, X.; Zhang, W.; Wu, H.; Yao, Z.; Atmos. Environ. 2018, 191, 249.
8. Bücker, F.; Santestevan, N. A.; Roesch, L. F.; Seminotti Jacques, R. J.; Peralba, M. do C. R.; Camargo, F. A. de O.; Bento, F. M.; Int. Biodeterior. Biodegrad. 2011, 65, 172.

9. Singh, S. P.; Singh, D.; Renewable Sustainable Energy Rev. 2010, 14, 200.

10. Rosa, H. A.; Wazilewski, W. T.; Secco, D.; Chaves, L. I.; Veloso, G.; de Souza, S. N. M.; da Silva, M. J.; Santos, R. F.; Renewable Sustainable Energy Rev. 2014, 38, 651.

11. Costa, E.; Almeida, M. F.; Alvim-Ferraz, C.; Dias, J. M.; Ind. Crops Prod. 2019, 129, 51.

12. de Aquino, G. S.; Hata, F. T.; Michelon, T. A.; Diniz, T. G.; Nicio, T. T.; de Oliveira, A. L. M.; Watanabe, V. S.; Ventura, M. U.; Alexandrino, R. P.; Pescador, P. G. de A.; Ind. Crops Prod. 2018, 121, 277.

13. Bondioli, P.; Folegatti, L.; Lazzeri, L.; Palmieri, S.; Ind. Crops Prod. 1998, 7, 231.

14. Onorevoli, B.; Machado, M. E.; Dariva, C.; Franceschi, E.; Krause, L. C.; Jacques, R. A.; Caramão, E. B. A.; Ind. Crops Prod.. 2014, 52, 8.

15. Lazzeri, L.; Leoni, O.; Conte, L. S.; Palmieri, S.; Ind. Crops Prod. 1994, 3,103 .

16. Zhu, L.-H. In Industrial Oil Crops; McKeon, T. A., Hayes, D. G., Hildebrand, D. F., Weselake, R. J., eds.; Elsevier: New York, 2016.

17. Jasper, S. P.; Biaggioni, M. A. M.; Silva, P. R. A.; Energia na Agricultura 2010, 25, 141.

18. Moser, B. R.; Fuel 2012, 99, 254.

19. Brasil, R. F. Lei $N^{o} .13 .263$, de 23 de Março de 2016. Altera a Lei $\mathrm{N}^{\circ}$ 13.033, de 24 de Setembro de 2014, Para Dispor Sobre Os Percentuais de Adição de Biodiesel Ao Óleo Diesel Co- Mercializado No Território Nacional. Diário Oficial da União 2016, 153. 93.

20. Kalligeros, S.; Zannikos, F.; Stournas, S.; Lois, E.; Energy 2003, 28, 15.

21. Corgozinho, C. N. C.; Pasa, V. M. D.; Barbeira, P. J. S.; Talanta 2008, $76,479$.

22. Mazivila, S. J.; Gontijo, L. C.; Santana, F. B. De; Mitsutake, H.; Santos, D. Q.; Borges Neto, W.; Energy Fuel 2015, 29, 227.

23. Nabi, M. N.; Rasul, M. G.; Energy Convers. Manage. 2018, 169, 326.

24. Meira, M.; Quintella, C. M.; Ferrer, T. M.; Gonçalves, H. R.; Guimarães, A. K.; Costa Neto, P. R.; Pepe, I. M.; Quim. Nova 2011, 34, 621.

25. EN 14078. Liquid Petroleum Products-Determination of Fatty Acid Methyl Ester (FAME) Content in Middle Distillates-Infrared Spectrometry Method. European Standard: BS EN - European Committee for Standardization: Bruxels, DC 2010.

26. ASTM Standard D7371; ASTM International 2014, 1.

27. Santana, F. B. de; Gontijo, L. C.; Mitsutake, H.; Mazivila, S. J.; Souza, L. M. de; Borges Neto, W.; Food Chem. 2016, 209, 228.

28. Mazivila, S. J.; Talanta 2018, 180, 239.

29. Ferrão, M. F.; Francesquett, J. Z.; Viera, M. de S.; Lovato, R. H.; Fachini, D.; Gerbase, A. E.; da Costa, A. B.; Tecno-Lógica 2010, 14, 87.

30. Coates, J. In Interpretation of Infrared Spectra, A Practical Approach; Meyer, R. A., ed.; New Town: Chichester, 2000.

31. Smith, B. C.; Fundamentals of Fourier Transform Infrared Spectroscopy, $2^{\text {nd }}$ ed., Taylor \& Francis Group: New York, 2011.

32. Skibsted, E. T. S.; Boelens, H. F. M.; Westerhuis, J. A.; Smilde, A. K.; Broad, N. W.; Rees, D. R.; Witte, D. T.; Anal. Chem. 2005, 77, 7103.

33. Rocha, W. F. de C.; Poppi, R. J.; Microchem. J. 2010, 96, 21.

34. Costa, F. S. L.; Pedroza, R. H. P.; Porto, D. L.; Amorimb, M. V. P.; Lima, K. M. G.; J. Braz. Chem. Soc. 2015, 26, 64.

35. Sullivan, J. H.; Woodall, W. H.; Journal of Quality Technology 1996, 28, 398.

36. Tôrres, A. R.; Grangeiro, S.; Fragoso, W. D.; Microchem. J. 2015, 118, 259.

37. de Oliveira, I. K.; de Carvalho Rocha, W. F.; Poppi, R. J.; Anal. Chim. Acta 2009, 642, 217.

38. Sitoe, B. V.; Máquina, A. D. V.; de Santana, F. B.; Gontijo, L. C.; Santos, D. Q.; Borges Neto, W.; Fuel 2017, 191, 299. 
39. Mitsutake, H.; Guimarães, E.; Freitas, H. C.; Gontijo, L. C.; Santos, D. Q.; Borges Neto, W.; J. Chemom. 2015, 29, 411.

40. Costa, E.; Almeida, M. F.; Alvim-Ferraz, M. da C.; Dias, J. M.; Renewable Energy 2018, 124, 27.

41. Atabani, A. E.; Mahlia, T. M. I.; Masjuki, H. H.; Badruddin, I. A.; Yussof, H. W.; Chong, W. T.; Lee, K. T.; Energy 2013, 58, 296.

42. Santos, D. Q.; Paula de Lima, A.; Martins Franco, M.; Maikel Fernandes, D.; Borges Neto, W.; Domingos Fabris, J.; AIMS Energy 2014, 2, 183.
43. Lin, J.-J.; Chen, Y.-W.; J. Taiwan Inst. Chem. Eng. 2017, 75, 43.

44. Lôbo, I. P.; Ferreira, S. L. C.; da Cruz, R. S.; Quim. Nova 2009, 32, 1596.

45. Lorber, A.; Faber, K.; Kowalski, B. R.; Anal. Chem. 1997, 69, 1620.

46. Miller, J.; Miller, J.; Statistics and Chemometrics for Analytical Chemistry, $5^{\text {th }}$ ed., Pearson Education/Prentice-Hall: Harrow, 2005.

47. Mudholkar, G. S.; Yu, Z.; Awadalla, S. S.; Statistics \& Probability Letters 2015, 107.

48. Jackson, J. E.; Mudholkar, G. S.; Technometrics 1979, 21, 341. 